\title{
Treatment of Typhoid Fever in Children: Comparison of Efficacy of Ciprofloxacin with Ceftriaxone
}

\author{
Dr. Amna Naveed \\ Pediatrics Specialist, Fellow College of Physicians and Surgeons Pakistan \\ Dr. Zeeshan Ahmed \\ Frontier Medical College, Pakistan
}

doi: 10.19044/esj.2016.v12n6p346 URL:http://dx.doi.org/10.19044/esj.2016.v12n6p346

\begin{abstract}
Objective: To compare the clinical efficacy of ciprofloxacin vs ceftriaxone in terms of the proportion of children becoming afebrile in 96 hours.

Study Design: Randomized controlled design study.

Place and Duration of Study: Department of Pediatrics, Holy Family Hospital, Rawalpindi from March 2010 to September 2010.

Methodology: 88 children who fulfilled the clinical criteria of having typhoid fever were included in the study. They came to the indoor and outdoor of the department. 44 patients were treated with injectable ciprofloxacin, while 44 were treated with injectable ceftriaxone.

Results: The study included 88 patients with febrile illness who were suspected of having typhoid fever. The mean age was $8.3 \pm 1.94$ years and 41 (46.6\%) were males. The mean weight was $24.7 \pm 6.3 \mathrm{~kg}$. Only $15(17 \%)$ used boiled water as a routine. 68 (77.3\%) children in total became afebrile within 96 hours and $20(22.7 \%)$ failed to become afebrile in 96 hours. In the ciprofloxacin group, 25 (56.8\%) patients became afebrile in 96 hours and 19 (43.1\%) failed to become afebrile in 96 hours. In the ceftriaxone group, 43 (97.7\%) patients became afebrile in 96 hours and 1 (2.3\%) failed to become afebrile in 96 hours. The proportion of patients becoming afebrile within 96 hours was significantly higher in the ceftriaxone group as compared to the ciprofloxacin group, $\mathrm{p}=0.00$.
\end{abstract}

Conclusion: Ceftriaxone is more effective in children with typhoid fever in terms of greater proportion of children becoming afebrile in 96 hours.

Keywords: Typhoid Fever, Ceftriaxone, Efficacy of drug 


\section{Introduction}

Typhoid fever, a communicable disease caused by the bacteria Salmonella typhoid, has been a major cause of significant morbidity and mortality since antiquity. Salmonellae are important gram-negative bacilli which cause a spectrum of characteristic clinical syndromes including gastroenteritis, enteric fever, bacteremia, endovascular infections, and focal infections such as osteomyelitis or abscesses. Enteric fever, also called typhoid fever or paratyphoid fever, is a systemic febrile illness that is most commonly caused by Salmonella typhi. Less frequently, it is caused by S. paratyphi A, S. typhi B, and S. paratyphi C. Even "nontyphoidal" Salmonellae may cause severe illness which is consistent with enteric fever. Complications are more common in untreated individuals and they include intestinal hemorrhage and perforation, or focal infection such as visceral abscesses. In the preantibiotic era, approximately 15 percent of afflicted individuals died, with survivors experiencing a prolonged illness lasting weeks and debilitation often-lasting months. Furthermore, approximately 10 percent of untreated individuals relapse, while 1 to 4 percent becomes chronic carriers of the organism.

Pakistan has the third highest incidence rate of typhoid occurring in the general population, worldwide. Typhoid fever is a common presentation in pediatric clinics. In the western world, the disease is close to eradication levels. However, globally, there are at least 13 to 17 million cases resulting in 600000 deaths. Typhoid fever represents the $4^{\text {th }}$ most common cause of death in Pakistan. It is transmitted by fecal oral root and by contamination of food and water. World Health Organization identifies typhoid as a serious public health problem. Its incidence is highest in children and young adults between 5-19 years old. WHO showed that the incidence of typhoid fever in Pakistani children aged 2-5 years was 573.2 per 100,000 persons per year. Also, similar incidence was seen in school going children and adolescents. The highest burden of disease is in children aged 2-15 years. Therefore, $S$ Typhi represents the commonest cause of bacteraemia in this age group, and annual typhoid rates (confirmed by blood culture) in recent studies from India, Pakistan, and Indonesia range from 149 to as high as 573 cases per 100000 children. The definitive diagnosis of typhoid fever is made only on isolation of salmonella typhi from blood, stool, urine, bone marrow etc, in the presence of characteristics clinical features. The case fatality ratio is $10 \%$ in the absence of treatment, and less than $1 \%$ with the use of antibiotics.

Fluroquinolones i.e. Ciprofloxacin, are recommended as $1^{\text {st }}$ line therapy for children and adults infected with sensitive as well as multidrug resistance, S. Typhi and paratyphi. Third generation cephalosporins i.e. Ceftriaxone, is also useful but their use is reserved for complicated cases Multidrug resistance (resistance to chloramphenicol, ampicillin, and co- 
trimoxazole) sequentially increased from 34\% in 1999 to $66 \%$ in 2005. In a prospective study in North India, there was a gradual development of resistance to fluoroquinolones over the past 7 years. No resistance was observed to fluoroquinolones in 1999, while in 2005, 4.4\% resistance was observed to sparfloxacin, $8.8 \%$ resistance to ofloxacin, and a high resistance, $13 \%$, to ciprofloxacin. Keeping in mind drug resistance in the community, it can be questioned that either of these drugs have difference in efficacy in terms of sensitivity and resistance pattern and relapse. In addition, we plan to conduct a study to find out the clinical response in children with typhoid fever treated with ciprofloxacin vs ceftriaxone. Thus, the results of the study will enable paediatricians to choose the first line therapy for the treatment of enteric fever in similar setting. In this way, timely management of these children will reduce morbidity specifically and also reduce the burden on hospital clinics in general.

\section{Methodology}

The study was conducted at Pediatric Department, Holy Family Hospital, Rawalpindi. Study was conducted over six months from 25th March 2010 to $24^{\text {th }}$ September 2010. A total of 88 patients with clinical diagnosis of typhoid fever were included in the study. 44 patients were treated with ciprofloxacin (Ciprofloxacin group), while 44 were treated with ceftriaxone (Ceftriaxone group).

\section{Consecutive (non-probability) Sampling Technique}

Children 5-12 years old of both sexes having typhoid fever were included in the study. All those having current history of intake of oral or I/V antibiotics (third generation cephalosporins and quinolones) and absence of fever at the time of presentation were excluded. However, this was a Randomized Controlled Trial study. Patients fulfilling the study criteria were admitted in the pediatric ward of Holy Family Hospital and were randomly divided into two groups, A and B, based on random numbers. Group A was given Inj.Ciprofloxacin 10mg/kg I/V twice daily, while group B was given Inj. Ceftriaxone $70 \mathrm{mg} / \mathrm{kg} \mathrm{I/V}$ once daily for 7 days. Both groups were observed for the duration of becoming afebrile (96hrs). The investigation to be done during hospital stay was typhidot (IgM antibodies) from the designated laboratory with the standard kit. However, the results were verified by the consultant pathologist. Data was analyzed using SPSS (V10). Mean and standard deviation were calculated for quantitative variables i.e. age and duration of getting afebrile. Frequencies and percentages were calculated for qualitative variables i.e. gender and getting afebrile in 96 hours. Consequently, chi-square test was used to compare the efficacy 
(afebrile in 96hours) of both drugs. P-value $<0.05$ was considered significant.

\section{Result}

The study included 88 patients with febrile illness suspected of having typhoid fever on clinical grounds. Clinically, the patients had fever $>37^{\circ} \mathrm{C}$ in the presence of at least one or more of the following sign and symptoms: Persistent headache, Abdominal pain or discomfort, Presence of splenomegaly / hepatomegaly, Rose spots on skin, vomiting, and no evidence of chest bowel urine or meningeal infection. Thus, all subjects were $<12$ years of age. The age range was from 5 to 12 years with a mean age of $8.3 \pm 1.94$ years. $41(46.6 \%)$ were males and 47 (53.4\%) were females. The weight of the children ranged from 14 to $41 \mathrm{~kg}$ with a mean weight of $24.7 \pm 6.3 \mathrm{~kg}$. Furthermore, 15 (17\%) used boiled water as a routine, while 73 (83\%) used unboiled water as a routine.

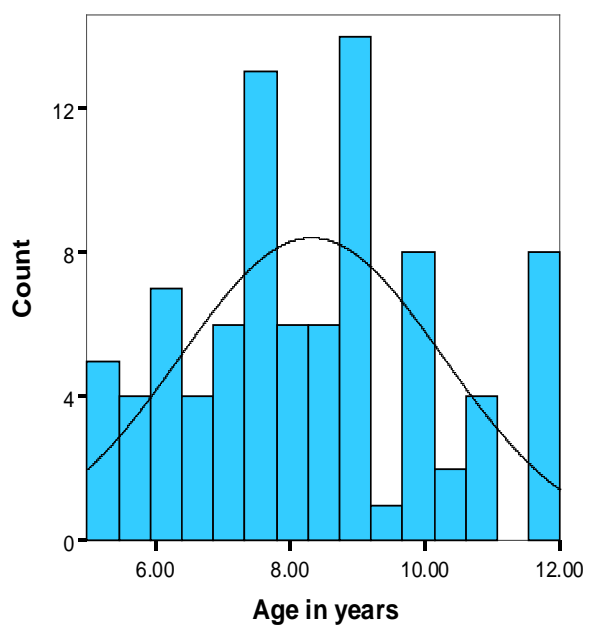

Figure No 1. Histogram Showing Age Distribution of Study Group 


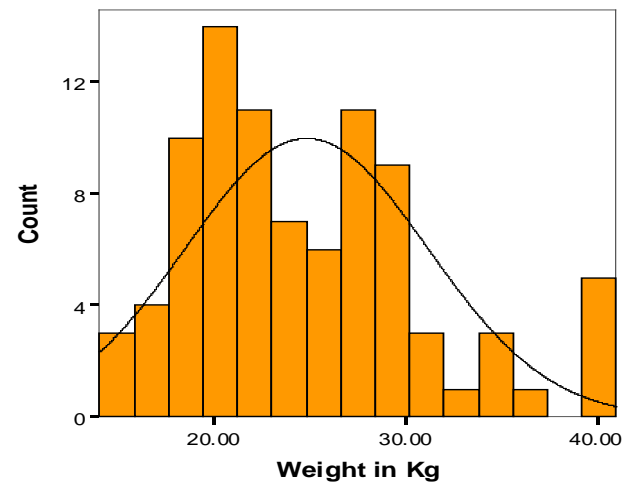

Figure No 2. Histogram Showing Weight Distribution of Study Group

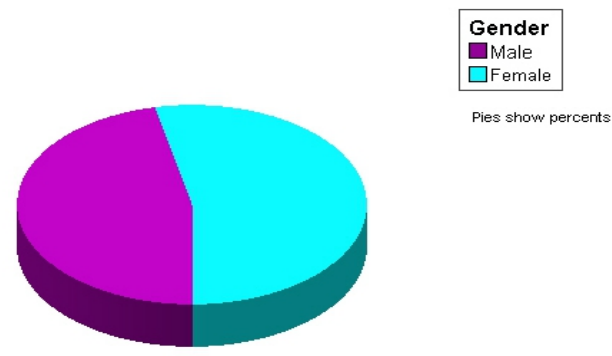

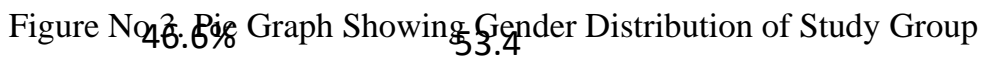

Table 1. Gender Distribution in Study Groups

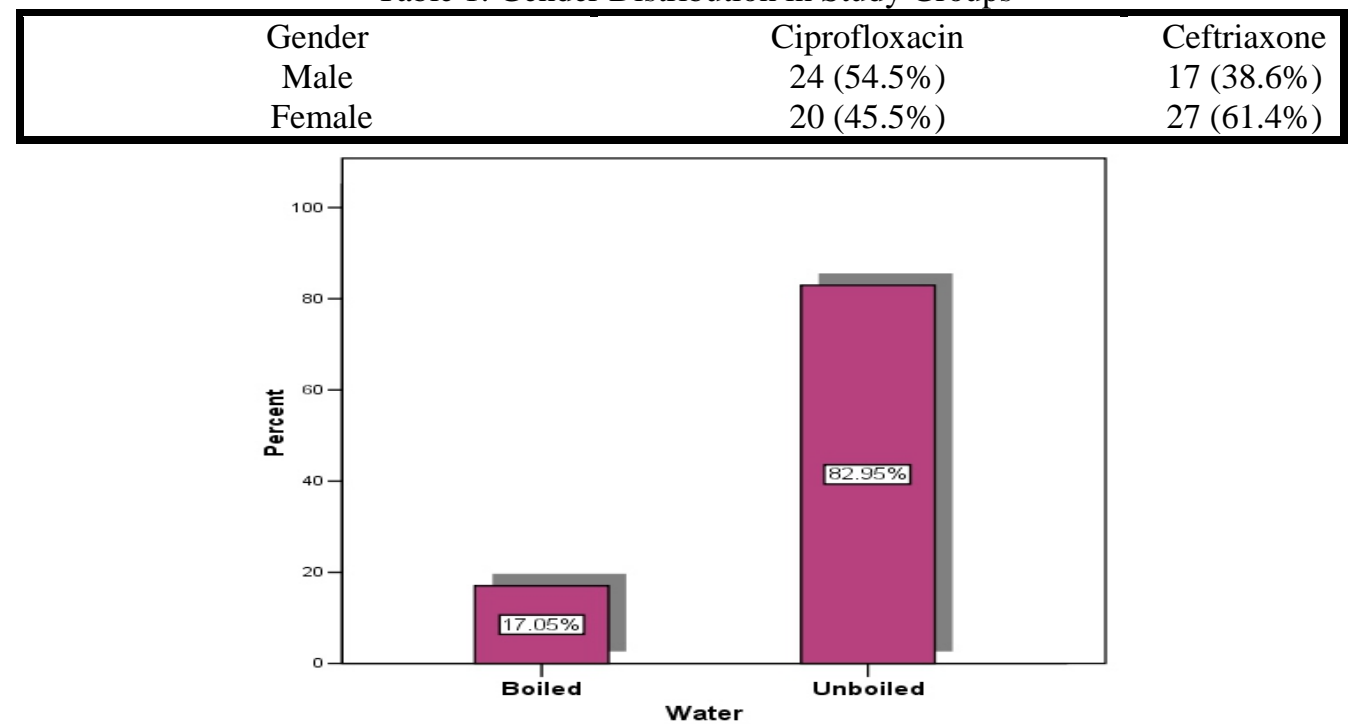

Figure No 4. Water used Boiled and Non-boiled 
Table 2. Chi square test comparing the frequency of patients becoming afebrile in 96 hours; ciprofloxacin versus ceftriaxone groups

\begin{tabular}{|cr|c|c|c|}
\hline & & \multicolumn{2}{|c|}{ Antibiotic group } & \multirow{2}{*}{ Total } \\
\cline { 3 - 4 } & & $\begin{array}{c}\text { Ciproflox } \\
\text { acin }\end{array}$ & Ceftriaxone & \\
\hline Afebrile in 96 hours & Yes 83\% & 25 & 43 & 68 \\
& No & 19 & 1 & 20 \\
Total & $17 \%$ & 44 & 44 & 88 \\
\hline
\end{tabular}

\begin{tabular}{|cccccc|}
\hline & Value & Df & Asymp. Sig. (2. & Exact Sig. & Exact Sig. \\
(2-sided) & (1-sided) \\
Pearson Chi-Square & $20.965(\mathrm{~b})$ & 1 & .000 & & \\
Continuity & 18.700 & 1 & .000 & & \\
Correction(a) & 24.607 & 1 & .000 & & \\
Likelihood Ratio & & & & .000 & .000 \\
Fisher's Exact Test & 88 & & & & \\
N of Valid Cases & & & & & \\
\hline
\end{tabular}

Chi-Square Tests

a Computed only for a $2 \times 2$ table

b 0 cells (.0\%) have expected count less than 5 . The minimum expected count is 10.00 .

\section{Discussion}

Enteric fever is a common illness of children and young adults. This magnifies the socio-economic impact of the disease on the community. The industrialized and more prosperous countries have, to a great extent, controlled this illness by improving standards of public health; but the disease continues to be a major public health problem in less developed countries including Pakistan. Therefore, the emergence of drug resistant strains of Salmonella has made the treatment of enteric fever more difficult. The last 2 decades have also witnessed the appearance and spread of multidrug-resistant (MDR) strains of S. typhi. Infection with these strains is associated with longer duration of illness and higher morbidity and mortality. Thus, it is occurring at higher incidence throughout South Asia than previously thought particularly in younger children. Enteric fever is a significant problem in the preschool years. Among children, $60 \%$ of cases are in the age group of 5 to 9 years, 27\% between 2-5 years, and $13 \%$ between age group of $0-2$ years. ${ }^{208}$ After the emergence of chloramphenicol resistant Salmonella typhi strains, ciprofloxacin has become the choice of drugs for the treatment of typhoid fever even in the pediatric age group.

The present study was carried out to compare the clinical efficacy of ciprofloxacin vs ceftriaxone in terms of the average time taken in the number of days for defevescence in the treatment of typhoid fever in children. 
Therefore, the study included 88 patients with febrile illness suspected of having typhoid fever. The mean age was $8.3 \pm 1.94$ years and 41 (46.6\%) were males. The mean weight was $24.7 \pm 6.3 \mathrm{~kg}$. Only 15 (17\%) used boiled water as a routine. $68(77.3 \%)$ children in total became afebrile within 96 hours, while $20(22.7 \%)$ failed to become afebrile in 96 hours. In the ciprofloxacin group, 25 (56.8\%) patients became afebrile in 96 hours and 19 (43.1\%) failed to become afebrile in 96 hours. In the ceftriaxone group, 43 (97.7\%) patients became afebrile in 96 hours and 1 (2.3\%) failed to become afebrile in 96 hours. Therefore, the proportion of patients becoming afebrile within 96 hours was significantly higher in the ceftriaxone group as compared to the ciprofloxacin group, $\mathrm{p}=0.00$.

Our results thus differ from those of previous review, a summary of randomised controlled trials of enteric fever, which found fluoroquinolones to be superior to ceftriaxone for clinical failure and fever clearance times. Although these data suggest that fluoroquinolones had significantly lower fever clearance times compared with chloramphenicol, cefixime, and ceftriaxone, the analyses of fever clearance times must be interpreted with caution. The mean fever clearance times often follow a skewed distribution —although most patients clear fever quickly, some take much longer times-, so meta-analyses conducted using arithmetic means may not be accurate. However, the persistence of fever in some patients despite apparent clearance of S Typhi and S Paratyphi from the bloodstream has been attributed to the continued production of pyrogenic cytokines. This suggests that time taken to clear fever may not be an adequate measure of antibiotic efficacy. Consequently, they may not be an appropriate end point in typhoid therapy trials. Some investigators also did not specify whether clinical failures were excluded or included in the calculations of mean fever clearance time. This may be due to the irrational use of quinolones even in minor infections. Hence, most of this includes viral fevers. This has resulted in increasing the resistance of $S$ typhi to quinolones in our country.

Appropriate treatment for enteric fever is a clinical and public health challenge, with rising levels of drug resistance and limited evidence for the use of newer agents, particularly for children. Large, well designed, and methodologically rigorous trials are needed to compare fluoroquinolones with first line antibiotics in community or outpatient settings. Hence, this reflects practice in low income countries, with accurate reporting of resistance data. Long term follow-up and monitoring of adverse effects are also required. Investigators must standardize definitions and time points of measurements of outcomes, particularly those with a subjective nature, such as clinical failure. In addition to objective studies of treatment efficacy and cost effectiveness, we need the evaluations of algorithmic approaches in the diagnosis and management of prolonged fever in children in regions where 
typhoid is endemic. Such protocols will guide antibiotic use and may curb rising resistance. A study at the Divisions of Clinical Medicine \& Microbiology, National Institute of Cholera \& Enteric Diseases (ICMR), Kolkata, India, evaluated the role of ceftriaxone therapy in bacteriologically confirmed MDR typhoid cases who did not respond to 12-14 days of ciprofloxacin therapy. Attempts have also been made to investigate the in vitro susceptibility of isolated S. typhi strains to chloramphenicol, ciprofloxacin, and ceftriaxone. A total of 140 children, aged 3-10 years, clinically diagnosed as having typhoid fever, without any clinical response after 12-14 days of ciprofloxacin therapy were screened for S. typhi by blood culture. In the bacteriologically positive children, the treatment was changed to intravenous ceftriaxone for 14 days. The isolated strains of S. typhi were tested for in vitro antimicrobial susceptibility. Clinical and bacteriological cure was observed with intravenous ceftriaxone therapy in all the 32 bacteriologically positive patients. All isolated S. typhi strains were uniformly (100\%) susceptible to ciprofloxacin and ceftriaxone. Thus, 50 percent of the strains were resistant to chloramphenicol. The MIC values of chloramphenicol, ciprofloxacin, and ceftriaxone ranged between 125-500, $0.0625-0.5$ and $<0.0625$ microgram $/ \mathrm{ml}$, respectively. The study indicates that although the S. typhi strains were susceptible to ciprofloxacin in vitro, the patients did not respond clinically and bacteriologically to ciprofloxacin therapy. Hence, ciprofloxacin may not represent a reliable and useful option for treating MDR typhoid fever; thus, ceftriaxone may be an effective alternative for the treatment of such cases.

Typhoid fever is widely prevalent in developing countries, with an annual burden of millions of cases globally. At Lady Reading Hospital, Peshawar, a study on drug resistance in enteric fever was carried out in Paediatric "A" Unit. Inclusion criterion was positive blood and/or bone marrow culture. In total, 50 patients had positive culture for salmonella (blood in 26 patients and bone marrow in 49 patients). Organism isolated were salmonella typhi in 49 cases and salmonella paratyphi A in one case. The single isolate of S paratyphi A was sensitive to all the antimicrobials tested except cotrimoxazole. Out of 49 isolates of S typhi, only 5 (10.2\%) were sensitive to all the primary anti-typhoid antimicrobials, while 44 (89.8\%) were resistant to multiple drugs. All of the isolates here were fully sensitive to ciprofloxacin and ofloxacin, while sensitivity to third generation cephalosporins varied between $57 \%$ and $79 \%$. Inspite of in vitro resistance, 22 patients (44\%) showed good clinical response to amoxycillin and chloramphenicol. In the remaining 28 patients (56\%), response to the above drugs was poor, and they were started on ofloxacin (in children above 5 years of age) or third generation cephalosporins. Consequently, the response of the patients to these drugs was good with defervescence within 8 days 
after the start of treatment No significant effects of quinolones were noted in these children. They concluded that quinolones can be used in children above 5 years of age in multidrug - resistant typhoid fever. The rapid spread of multidrug resistant (MDR) typhoid fever has posed a great challenge for the treatment of these cases all over the world today. After the emergence of chloramphenicol resistant Salmonella typhi strains, ciprofloxacin has become the drug of choice for the treatment of typhoid fever even in the paediatric age group. Thus, study at Kolkata, India, evaluated the role of ceftriaxone therapy in bacteriologically confirmed MDR typhoid cases that did not respond to 12-14 days of ciprofloxacin therapy. They included 140 children aged 3-10 years. Therefore, they found that ciprofloxacin might not represent a reliable and useful option for treating MDR typhoid fever. In addition, ceftriaxone may be an effective alternative for the treatment of such cases. ${ }^{i}$

\section{Conclusion}

Ceftriaxone is more effective in children with typhoid fever in terms of greater proportion of children becoming afebrile in 96 hours

\section{References:}

Acharya G, Butler T, Ho M, Sharma PR, Tiwari M, Adhikari RK, et al. (1995). Treatment of typhoid fever: randomized trial of a three-day course of ceftriaxone versus a fourteen-day course of chloramphenicol. Am J Trop Med Hyg;52:162-5

Bhutta Z A (2006). Current concepts in the diagnosis and treatment of typhoid fever. BMJ.333:78-82

Bhutta Z A, Naqvi SH, Razzaq RA, Farooqui BJ (1991). Multidrug-resistant typhoid in children: presentation and clinical features. Rev-Infect-Dis; 13: 832-6.

Butler, T. (2011). Treatment of typhoid fever in the 21st century: promises and shortcomings. Clinical Microbiology and Infection, 17: 959-963. doi: 10.1111/j.1469-0691.2011.03552.

Dutta P, Mitra U, Dutta S, De A, Chatterjee MK, Bhattacharya SK (2001). Ceftriaxone therapy in ciprofloxacin treatment failure typhoid fever in children. Indian J Med Res 2001;113:210-3.

Gandapur AJ, Khan FR, Zeb A, Khan FM, Imran M (1993). A Study of 100 patients with enteric fever in children, at peshawar. PPJ 1993;17:19-25

Hay WW, Levin MJ, Sondheimer JM (2009). Typhoid fever and Para Typhoid fever In: Current Diagnoses and Treatment Pediatrics. $19^{\text {th }}$ edition. 2009; pp1154-6

Imran M, Khan FR, Khattak AA, Aurang Zeb, Liaqat Ali L (1996). Multi drug - resistant Enteric Fever in children Pak Paed J 1996;20:169-73. 
Kumar S, Rizvi M, Berry N (2008). Rising prevalence of enteric fever due to multidrug-resistant Salmonella: an epidemiological study. J Med Microbiol 2008; 57(Pt 10):1247-50.

Mandal BK (1990). Treatment of Multriresistant typhoid fever (letter). Lancet 1990;339:1383.

Ochiai RL, Acosta CJ, Danovaro-Holiday MC, Bai-Ging D, Bhattacharya SK, Agtini MD et al. (2008). A Study of Typhoid Fever in time Asian countries: Disease burden in implication for control. Bull World Health Org 2008; 86:241-320.

Rafiq H, Zia R, Naeem S (2009). Typhoid fever - continues as a major threat in children. Biodemica 2009; 25: 1-2.

Siddiqui FJ, Rabbani F, Hasan R, Nizami SQ, Bhutta ZA (2006). Typhoid fever in children: some epidemiological considerations from Karachi, Pakistan. Int J Infect Dis 2006;10:215-22.

Siddiqui S (1991). Epidemiologic patterns and control strategies in typhoid fever. JPMA 1991;41:143-6.

Sinha A, Sazawal S, Kumar R, Sood S, Reddaiah VP, Singh B, et al. (1999). Typhoid fever in children aged less than 5 years. Lancet 1999; 354:734-7.

Stern JM, Simes RJ (1997). Publication bias: evidence of delayed publication in a cohort study of clinical research projects. BMJ 1997;315:640-5.

Thaver D, Zaidi AKM, Critchley J, Azmatullah A, Madni SA, Bhutta ZA (2009). A comparison of fluoroquinolones versus other antibiotics for treating enteric fever: Meta-analysis. BMJ 2009; 338: b1865.

Verma M, Parashar Y, Singh A, Kamoji R (2007). Current pattern of enteric fever: a prospective clinical and microbiological study. J Indian Med Assoc 2007;105:582-4.

World Health Organization (2006). $6^{\text {th }}$ International Conference on typhoid Fever and other Solmonelloses. WHO Geneva 2006. 